\title{
Modelling of Surface Gap State Passivation and Fermi Level De-pinning in Solar Cells
}

\author{
H Lu, Y Guo2, H Li, J Robertson*, \\ Engineering Dept, Cambridge University, Cambridge CB3 0FA, UK \\ 2 Swansea University, Swansea SA2 8PP, UK \\ * jr@eng.cam.ac.uk
}

\begin{abstract}
The behaviour of gap states due to coordination defects (eg dangling bonds) and metal induced gap states (MIGS) are compared using density functional supercell calculations. Whilst both types of gap states cause carrier recombination, they are passivated in different ways. Defects can be passivated by shifting their states out of the gap, whereas MIGS lie on normally coordinated atoms and their states cannot be shifted. Their 'passivation' requires the insertion of an insulating layer to attenuate them sufficiently before they enter the semiconductor. We show that MIGS also cause Fermi level pinning, inhibiting the control of work function by the contacts, and so they must also be attenuated to enable certain solar cell designs.
\end{abstract}

Recombination losses at the contacts are now a major factor limiting Si solar cell efficiencies [1]. This leads to a focus on contact passivation and thus it is critical to understand the behaviour of the gap states causing recombination. At the same time, especially for $\mathrm{Si}$, there is a desire to reduce production costs, for example by using lower processing temperatures or lower quality $\mathrm{Si}[2,3]$. An example is a trend to define carrier-selective contacts not by doping but by introducing contacts of unusually high or low work function. Both of these aspects require a clear understanding of the passivation of gap states in PV devices and their interfaces.

Recombination can be caused by two types of gap states, those due to mis-coordinated atoms such as Si dangling bonds (DBs), and those due to the effect of metals or transparent conducting oxide layers which are called metal-induced gap states (MIGS). We will show how each type requires different mitigation methods in order to reduce recombination losses.

Dangling bonds are typically at $\mathrm{Si}$ vacancies, $\mathrm{Si} /$ oxide interfaces or in amorphous (a-) $\mathrm{Si}: \mathrm{H}$ networks [4]. A single Si DB is an under-coordinated site and it can be passivated, for example, by a simple chemical reaction with hydrogen, as shown in the schematic density of states diagram of Fig 1(a) $[5,6]$. This converts the gap states of the DB into Si-H bonding and anti-bonding states lying outside the band gap, in the valence and conduction bands, respectively. This is the conventional process of defect passivation.

A bare Si surface possesses dangling bonds. If a metal layer lies on top of it, the Si DBs interact with the metal states to form MIGS [7]. The MIGS are extensions of the metal's travelling wave states continuing as evanescent states into the semiconductor band gap, Fig 1(c). The MIGS extend across the whole gap [7-9]. They are occupied up to the charge neutrality level (CNL) on a neutral surface. The surface MIGS density can in principle greatly outnumber the defect density, because there is one MIGS for every surface atom [8].

The surface Si DBs can be terminated by hydrogen or oxygen atoms. This removes their gap states when they are on a bare surface. But it does not remove the MIGS if the surface is covered with a metal. The MIGS originate as extensions of the metal states. Once in the Si, MIGS lie on fully bonded Si atoms [7,9], so their energy cannot be shifted out of the gap by a passivation reaction like DB states can. They can only be attenuated more rapidly by inserting a layer of wider band gap material (eg $\mathrm{Al}_{2} \mathrm{O}_{3}, \mathrm{SiO}_{2}$, $\mathrm{HfO}_{2}, \mathrm{MgO}, \mathrm{Si}_{3} \mathrm{~N}_{4}$ etc) so they have smaller amplitude when they enter the Si itself, Fig 1(d), [10,11].

DBs and MIGS are both amphoteric gap states with $-1,0$, and +1 charge states, so they can both act as recombination centers. Thus, MIGS and defect state densities must both be reduced to lower the recombination rates to make a 'passivated' contact. 
The gap states have a second property relevant to contact behavior. Both defect and MIGS states add up to give a total density of states which can cause Fermi level pinning (FLP). FLP limits the degree to which the surface Fermi level of a semiconductor can be shifted across the gap by varying the work function of the metal contact $\Phi_{\mathrm{m}}$. The surface Fermi level is measured by the n-type Schottky barrier height $(\mathrm{SBH}), \phi_{\mathrm{n}}$, from the conduction band minimum (CBM),

$\phi_{\mathrm{n}}=\mathrm{S} \Phi_{\mathrm{m}}+\mathrm{b}$

where $\mathrm{b}$ is a constant. The degree of FLP is given by the slope factor $\mathrm{S}=\partial \phi_{\mathrm{n}} / \partial \Phi_{\mathrm{m}}$, where $\mathrm{S}$ is given by

$$
S=\frac{1}{1+\frac{N e \delta}{\varepsilon_{\infty}}}
$$

in the Cowley-Sze model [12]. Here, $\mathrm{N}$ is the total density of gap states, e is the electronic charge, $\delta$ is the width of the interfacial layer and $\varepsilon$ is the dielectric constant. Large $\mathrm{N}$ and $\mathrm{S} \sim 0$ corresponds to a large Fermi level pinning (Bardeen limit), while small $\mathrm{N}$ and $\mathrm{S} \sim 1$ corresponds to weak FLP (Schottky limit), Fig 1(b). Pinning limits the ability to vary the contact SBH by varying the metal, and thus lower the contact resistance. Thus, weak FLP is an advantage in this context. Hence low recombination rates and weak FLP are both valuable and sometimes these two factors are not distinguished.

The strong FLP at a direct Si/metal interface can be mitigated by inserting a wider-gap layer (eg oxide) between the metal and the semiconductor, Fig 1(d). The layer acts like a wide-gap semiconductor [9-11] whose MIGS decay rate $\mathrm{k}$ varies [13] with

$\mathrm{k}\left(\AA^{-1}\right)=\mathrm{c} .(\text { band offset } \mathrm{x} \text { tunnelling mass })^{1 / 2}$

The MIGS decay more quickly inside this layer, emerging attenuated at the insulator/Si interface, so their pinning effect via eqn. (2) is much less.

The role of MIGS in FLP in PVs was recently briefly covered by Robertson [14] and in recombination by Sajjad et al [15]. These effects are now confirmed by more detailed density function supercell calculations. Fig 2(a) shows a bare $\mathrm{Si}(100)$ surface terminated by oxygens, denoted Si:O. A symmetric bridge structure reconstructs into one where an oxygen bonds to a single $\mathrm{Si}$ site as a $\mathrm{Si}=\mathrm{O}$ group. Fig 3(a) shows the density of states (DOS) of this surface. The $\mathrm{O}$ termination removes all gap states below $0.8 \mathrm{eV}$, leaving some empty states above $0.8 \mathrm{eV}$. Fig 2(b) shows the unterminated $\mathrm{Si}(100)$ surface with a metal over-layer (using Ir for convenience). Fig 3(b) shows the partial density of states (PDOS) for this surface with MIGS lying across the whole gap and decaying quite slowly with depth into the Si layer.

Fig 2(c) shows the O-terminated $\mathrm{Si}(100)$ surface now with a metal overlayer on top. The $\mathrm{O}$ has relaxed from that in Fig. 2(a) back to the symmetric structure because of extra bonding to metal atoms. Fig. 3(c) shows the PDOS for this interface, at increasing depth into the Si. We see that the metal states have completely penetrated the oxygen monolayer, and their PDOS decreases only gradually into the Si. In fact, the PDOS is not much less than for an interface with a direct metal/Si contact, Fig 3(b). Thus monolayer oxygen termination has little blocking effect on MIGS compared to the unterminated case.

Fig 2(d) shows a $\mathrm{Si}(100)$ surface with two monolayers of cubic $\mathrm{HfO}_{2}$ placed on top. $\left(\mathrm{HfO}_{2}\right.$ is a representative insulator for the calculations, as it has a cubic phase with a reasonable lattice match to $\mathrm{Si}$.) On top of this is placed the metal slab. Fig 3(d) shows the gap state PDOS vs Si layer number for this structure. We see that the metal has again created MIGS in the band gap of the underlying Si, but these start in the Si at a much lower intensity than they do in Fig 3(c). They then exit the oxide and decay in the $\mathrm{Si}$ at the slower rate typical of Si states.

Fig 4 summarises the results of Fig. 3, how the MIGS intensity at $0.3 \mathrm{eV}$ above the valence band edge varies with layer depth below the top Si interface. There are two key results; (1) MIGS occur in all cases where there is a metal overlayer, Fig 3(b-d). (2) the presence of a Si:O termination in Fig 2(c) makes little difference to the MIGS in the Si when there is a metal layer present, despite the O removing any gap states of Si DBs when no metal overlayers are present. Thus, the O termination may passivate the Si 
surface in a chemical sense. But it does not passivate the MIGS. It is only when layers of $\mathrm{HfO}_{2}$ or a similar insulator are placed between the metal and Si that the MIGS intensity can be sufficiently reduced, by a factor of $\sim 12$ per monolayer, Fig. 4 . This effect will reduce recombination rates via the MIGS. A similar effect will occur for other insulators like $\mathrm{Al}_{2} \mathrm{O}_{3}, \mathrm{MgO}, \mathrm{SiO}_{2}$ or $\mathrm{Si}_{3} \mathrm{~N}_{4}$. To reduce recombination velocity from the bare value of $\sim 10^{7} \mathrm{~cm} / \mathrm{s}$ to $10^{2} \mathrm{~cm} / \mathrm{s}$ would require a MIGS reduction by $10^{5}$ or $4-5$ monolayers of oxide from Fig. 4 or $\sim 2.0-2.2 \mathrm{~nm}$, which is similar to that seen experimentally [16].

We now consider the effect of MIGS on Fermi level pinning and the S factor of eqn (2). We calculate $\mathrm{S}$ directly from these supercell models of $\mathrm{Si}$ with various numbers $\mathrm{HfO}_{2}$ monolayers and slabs of various metals on top, calculating the slope of the Schottky barrier height (SBH) to Si vs. metal work function in each case as in Fig 5(a). The p-type SBH $\phi_{\mathrm{p}}$ is given by the Si valence band maximum (VBM) energy to the metal Fermi energy at the interface. The Si VBM is found from the density of states of a middle Si layer. Fig 5(a) plots $\phi_{p}$ vs experimental value of metal work function [17], and $S$ is found as the slope of this line, from eqn (1).

Fig 5(a) shows the case of zero, one and two monolayers of inserted $\mathrm{HfO}_{2}$. Fig 5(b) shows the extrapolated variation of $\mathrm{S}$ vs. $\mathrm{HfO}_{2}$ thickness for thicker films, using $\mathrm{S}$ for bulk $\mathrm{HfO}_{2}$ from previous work [18]. The factor $(1-1 / \mathrm{S})$ is plotted as it is proportional to $\mathrm{N}$. We see that two layers of $\mathrm{HfO}_{2}(\sim 1 \mathrm{~nm})$ will unpin $\mathrm{E}_{\mathrm{F}}$ sufficiently, but 3 layers is needed to reach an unpinned case similar to the bulk. The required thickness experimentally [3,16,19] will vary with the band gap and band offset of the actual insulator [9,13], as in eqn (3). Experimentally, larger thicknesses $(10 \mathrm{~nm})$ of hole passivation layers are needed [3], perhaps because other factors are also involved.

Fermi level pinning has significant implications for device design. The dopant-free asymmetric selective contacts concept was recently proposed as a way to avoid the high temperature processing costs associated with doping [20,21]. The traditional Si solar cell design uses p-n junctions to create an asymmetry to collect the photo-generated carriers at opposite contacts. On the other hand, cells without explicit doping would use two contacts with a large difference of work functions to extract the photogenerated carriers, the so-called dopant-free asymmetric contact heterojunction (DASH) cells.

The band alignments for such DASH cells are similar to those in organic photovoltaic (OPV) cells. This band design works for OPVs because organic semiconductors have van der Waals bonds between molecules and between them and the contacts. Thus their band alignments follow roughly the electron affinity rule, as in Fig 6(a) $[22,23]$. In this case, the difference in surface potentials $\Delta \Phi_{\text {eff }}$ at each contact is roughly the difference in the work functions of the isolated contacts, $\Delta \Phi$. On the other hand, if the intervening semiconductor is $\mathrm{Si}$, with its strong FLP, the effective surface potential at each metal contact becomes pinned towards the Si charge neutrality level, and the difference is much smaller $[8,9], \Delta \Phi_{\text {eff }}=$ $\mathrm{S} . \Delta \Phi \sim 0.07 \mathrm{eV}$, Fig $6(\mathrm{~b})$. This difference is less that the splitting of the quasi-Fermi levels and the cell voltage is low. To stop this, the contacts must be unpinned by inserting suitable insulating layers, to increase $\mathrm{S}$ to at least $\mathrm{S} \sim 0.5$ in which case $\Delta \Phi_{\text {eff }}=\mathrm{S} . \Delta \Phi \sim 1 \mathrm{eV}$.

Typical contacts for such cells are as follows. A suitable anode contact is oxygen deficient $\mathrm{MoO}_{3-\mathrm{x}}$ [24], which has a very high work function. Oxygen deficiency makes $\mathrm{MoO}_{3-\mathrm{x}}$ a n-type semiconductor [24,25]. There are various possibilities for a cathode. $\mathrm{Al} / \mathrm{LiF}$ was used in OLEDs [22] and is a suitable n-type contact for photovoltaics, and there are various others such as inhomogeneous $\mathrm{MgO} / \mathrm{Al}$ layers [21] or nanostructured $\mathrm{Zn}$ silicate [26].

The unpinning effect of a few inserted oxide layers is balanced for thicker layers by the lower tunnelling probability and higher resistance, leading to an optimum oxide thickness [27]. Interestingly, our results find that roughly two layers of $\mathrm{HfO}_{2}$ or $0.7 \mathrm{~nm}$ is sufficient to unpin $\mathrm{E}_{\mathrm{F}}$, consistent with the 1.0 $\mathrm{nm}$ thickness found for $\mathrm{MgO} / \mathrm{Al}$ layers [21]. On the other hand, thicker insulating layers are needed to attenuate MIGS sufficiently to reduce recombination velocities enough [16]. Finally, when transparent conducting oxides (TCOs) are used as contacts, we note that MIGS only occur in energy ranges where there are continuum states on the other side of the interface [7,28]. This is across the whole gap for metal interfaces, but not for TCOs. 
In summary, the methods to passivate defect states and attenuate metal induced gap states to minimise their effects on surface recombination velocity and Fermi level pinning are calculated via supercell calculations. It is shown that Fermi level pinning should be countered in order to use high and low work function contacts with dopant-free cell designs.

Methods. The electronic structure of various metal overlayers on $\mathrm{Si}(100)$ were calculated in supercell models. The models had between 32 and 120 atoms. The supercells have 5-6 layers of metal, 8 bilayers of $\mathrm{Si}$, and 1, 2 or 4 monolayers of $\mathrm{HfO}_{2}$. The $\mathrm{HfO}_{2}$ is lattice-matched to $\mathrm{Si}$ if its cell is rotated by $45^{\circ}$. Each interface has two oxygen atoms per Si or Hf atom [29]. The bottom layer of $\mathrm{Si}$ is terminated by hydrogen. Ir metal is used for the calculations of Figs 2, 3. Finally there is a $15 \AA$ of vacuum gap separating the slabs from their periodic repeat. For Fig 5, where different metals are used, we keep the lateral supercell size as small as possible. Some metals such as $\mathrm{Ir}, \mathrm{Ru}, \mathrm{Ni}$ or Co lattices are close to $\mathrm{Si}$ and a $1 \mathrm{x} 1$ supercell is possible. In other cases, the metal lattice is rotated to give a commensurate cell. The plane-wave density functional code CASTEP is used [30], with ultra-soft pseudopotentials, a plane wave cut off energy of $340 \mathrm{eV}$ and the generalized gradient approximation (GGA) for the electron functional. A 4x4x1 Monkhorst-Pack k-point mesh is used for the $1 \times 1$ cells. For Sc, a 2x2x1 k point mesh is used. For $\mathrm{MoO}_{3}$, a $4 \times 2 \times 1 \mathrm{k}$ point mesh is used. All structures are relaxed to a residual force of under $10^{-5} \mathrm{eV} /$ atom.

Acknowledgements The authors gratefully acknowledge EPSRC EP/P005152/1, the China Scholarship Council and Prof Jin Jang of Kyung Hee University, Seoul.

\section{References}

1. J Melskens, B W H van de Loo, B Macco, L E Black, S Smit, W M M Kessels, IEEE J Photovoltaics 8373 (2018)

2. C Battaglia, A Cuevas, S DeWolf, Energy Environ Sci 91552 (2015)

3. A Cuevas, Y Wan, D Yan, C Samundsett, T Allken, X Zhang, Solar Ener Mats Solar Cells 18438 (2018)

4. S Olibet, E V Sauvain, C Ballif, Phys Rev B 87035326 (2007)

5. E Cartier, J H Stathis, D A Buchanan, App Phys Lett 631510 (1993)

6. W B Jackson, C C Tsai, S M Kelso, J Non-Cryst Solids 77281 (1985)

7. J Tersoff, Phys Rev Lett 52465 (1984)

8. W Mönch, Phys Rev Lett 581260 (1987)

9. J Robertson, J Vac Sci Technol B 181785 (2000); J Vac Sci Technol A 31050821 (2013)

10. T Nishimura, K Kita, A Toriumi, Appl Phys Exp 1051406 (2008)

11. M Kobayashi, A Kinoshita, K Saraswat, H S P Wong, Y Nishi, Tech Digest VLSI (2008) p54;

12. A W Cowley, S M Sze, J App Phys 363212 (1965)

13. Y C Yeo, T J King, C M Hu, App Phys Lett 812091 (2002)

14. J Robertson, Mat Res Soc symposium (Nov 2017)

15. M Sajjad, X Yang, P Altermatt, N Singh, U Schwingenschlogl, S DeWolf, App Phys Lett 114071601 (2019)

16. J Bullock, D Yan, A Cuevas, Phys Stat Solidi RRL 7946 (2013)

17. H B Michaelson, J App Phys 484729 (1977)

18. K Y Tse, J Robertson, Phys Rev Lett 99086805 (2007)

19. J Schmidt, RR Peibst, R Brendel, Sol Energy Mat Solar Cells 18739 (2018)

20. J Bullock, M Hettick, J Geissbuhler, A J Ong, T Allen, C M Sutter-Fella, T Chen, H Ota, E W Schalter, S DeWolf, C Ballif, A Cuevas, A Javey, Nature Energy 115031 (2016)

21. Y Wan, C Samundsett, J Bullock, M Hettick, T Allen, D Yan, J Peng, Y Wu, J Cui, A Javey A Cuevas, Adv Energy Mats 71601863 (2017)

22. S Braun, W R Salaneck, M Fahlmann, Adv Mater 211450 (2009);

23. J Hwang, A Wan, A Kahn, Mat Sci Eng R 641 (2009)

24. C Battaglia, S M deNicolas, S DeWolf, X Yin, M Zheng, C Baliff, A Javey, App Phys Lett 104113902 (2014)

25. Y Guo, J Robertson, App Phys Lett 105222110 (2014); J Meyer, P R Kidambi, B C Beyer, C Weijtens, A Kuhn, A Centeno, J Robertson, S Hofmann, Sci Rep 45380 (2014)

26. N Nakamura, J Kim, H Hosono, Adv Electron Mater 41700352 (2018)

27. A Agrawal, N Shukla, K Ahmed, S Datta, App Phys Lett 101042108 (2012)

28. J Robertson, App Phys Lett 94152104 (2009)

29. P W Peacock, K Xiong, K Tse, J Robertson, Phys Rev B 73075328 (2006); K Y Tse, D Liu, J Robertson, Phys Rev B 81035325 (2010)

30. S. J. Clark, M. D. Segall, C. J. Pickard, P. J. Hasnip, M. I. J. Probert, K. Refson, M. C. Payne, Zeitschrift Für Krist. 220, 567 (2005) 
Fig. 1. The two different passivation mechanisms. (a) Shifting the energy of a defect gap state (eg Si dangling bond) by reaction with hydrogen or oxygen. (b) Schematic of Fermi level pinning and slope factor, S. (c) MIGS at a metal -semiconductor interface, and after passivation of MIGS by causing them to decay faster by inserting a thin insulator overlayer.

Fig. 2 (a). Si(100) surface passivated by oxygens, denoted $\mathrm{Si}$ O. (b) $\mathrm{Si}(100)$ with metal overlayer on top, (c) $\mathrm{Si}(100)$ passivated by oxygen monolayer, but with a metal overlayer on top, denoted Si:O:metal. Note the $\mathrm{O}$ site becomes symmetric now. (The top layer $\mathrm{Si}$ and $\mathrm{O}$ sites in the most stable configuration are over-coordinated due to two partial bonds to metal sites.) (d) $\mathrm{Si}(100)$ with two monolayers of $\mathrm{HfO}_{2}$ and a metal overlayer on top, denoted $\mathrm{Si}: 2 \mathrm{~L}-\mathrm{HfO}_{2}$ :metal.

Fig. 3. (a) PDOS on $\mathrm{Si}$ atomic layers for O passivated $\mathrm{Si}(100)$ surface. Note the zero DOS in the band gap. (b) PDOS for the Si:metal structure. (c) PDOS for the Si:O:metal structure. (d) PDOS for the Si:2 layer $\mathrm{HfO}_{2}$ :metal structure. Note the much lower gap state PDOS for (d). Although density functional theory traditionally under-estimates the band gap of semiconductors, the quantum confined of the finite thickness supercell leads to a Si band gap larger than $1.1 \mathrm{eV}$.

Fig. 4. Decay of Si PDOS at E= $0.3 \mathrm{eV}$ vs Si bilayer number (2.7 $)$ ) on (100). Note that MIGS easily penetrate the top $\mathrm{O}$ passivation layer, and they need the $\mathrm{HfO}_{2}$ layers (blue) to strongly reduce the MIGS intensity.

Fig. 5. (a) Calculated SBH vs metal work function for 0,1 or 2 monolayers of $\mathrm{HfO}_{2}$ on $\mathrm{Si}(100)$. (b) schematic of variation of slope factor vs number of $\mathrm{HfO}_{2}$ layers.

Fig.6. (a,b) Schematic of the effect of unpinned and pinned Fermi levels on the band alignments of some transparent conducting contacts on $\mathrm{Si}$, and their Schottky barrier heights. 

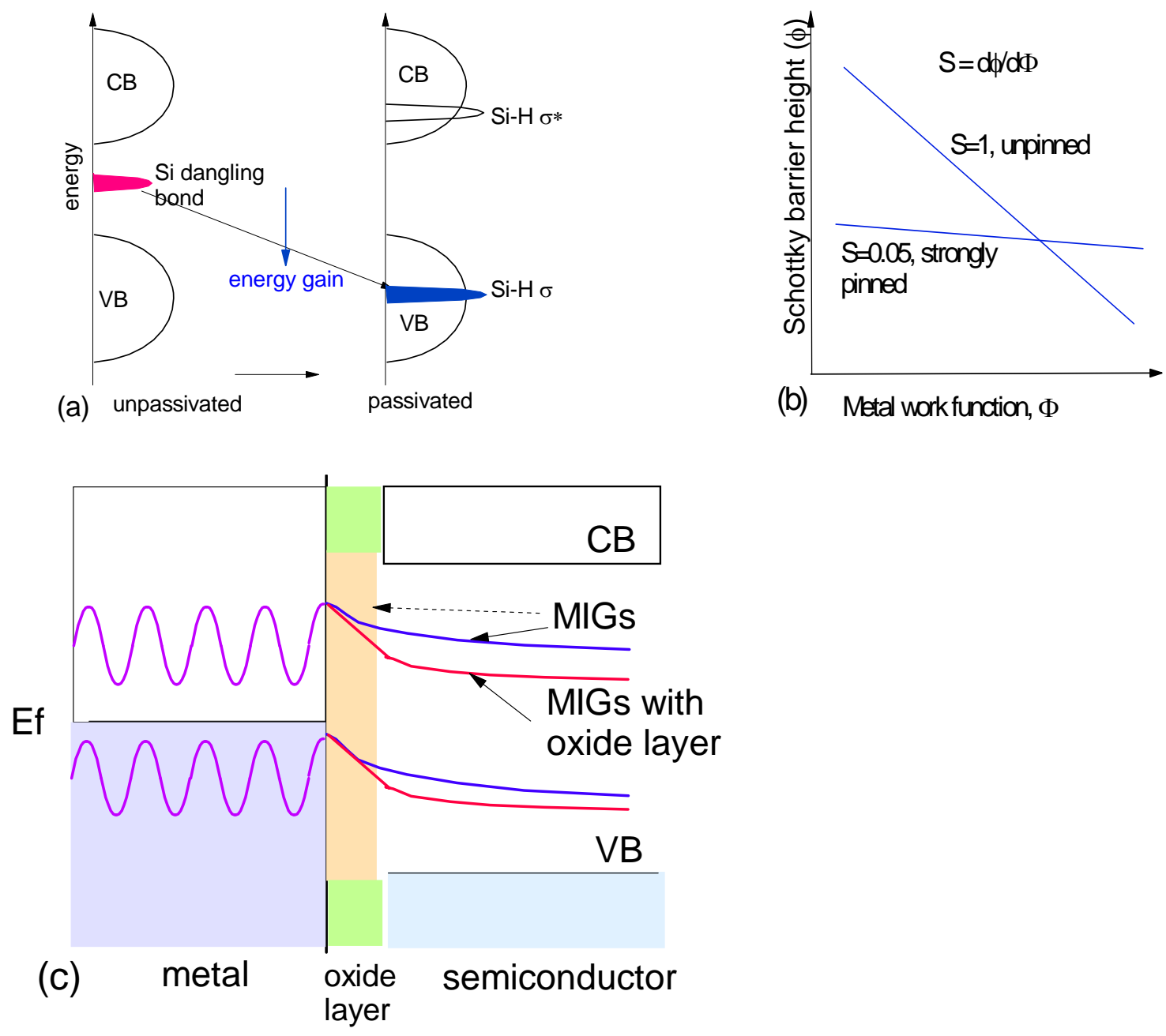

Fig. 1. 


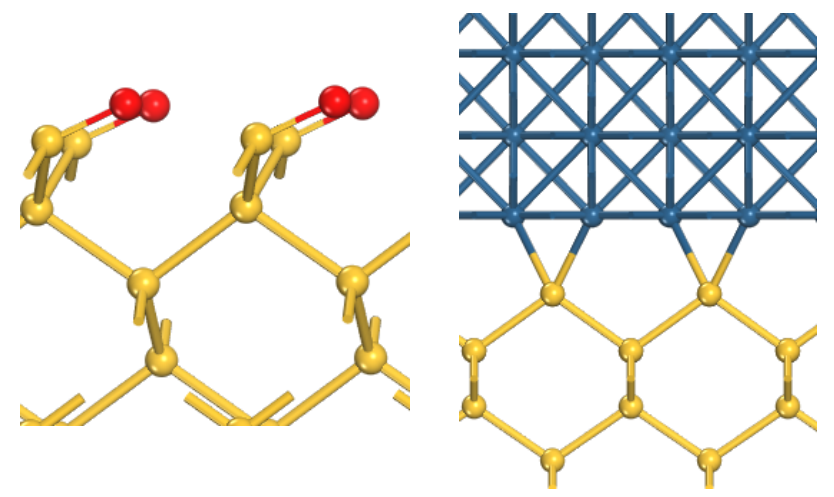

(a)

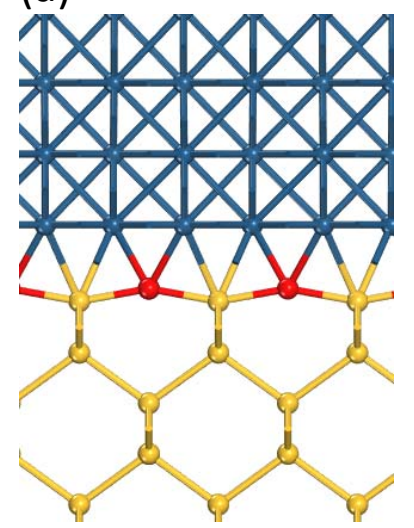

(c)

(b)

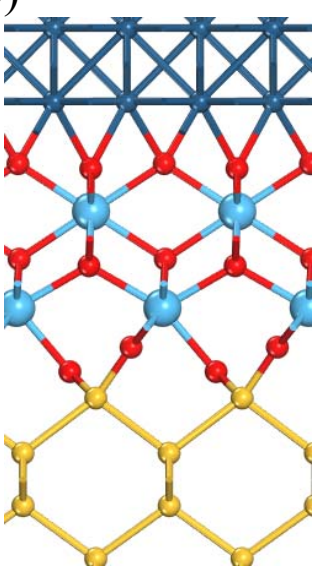

(d)

Fig. 2 
(a)
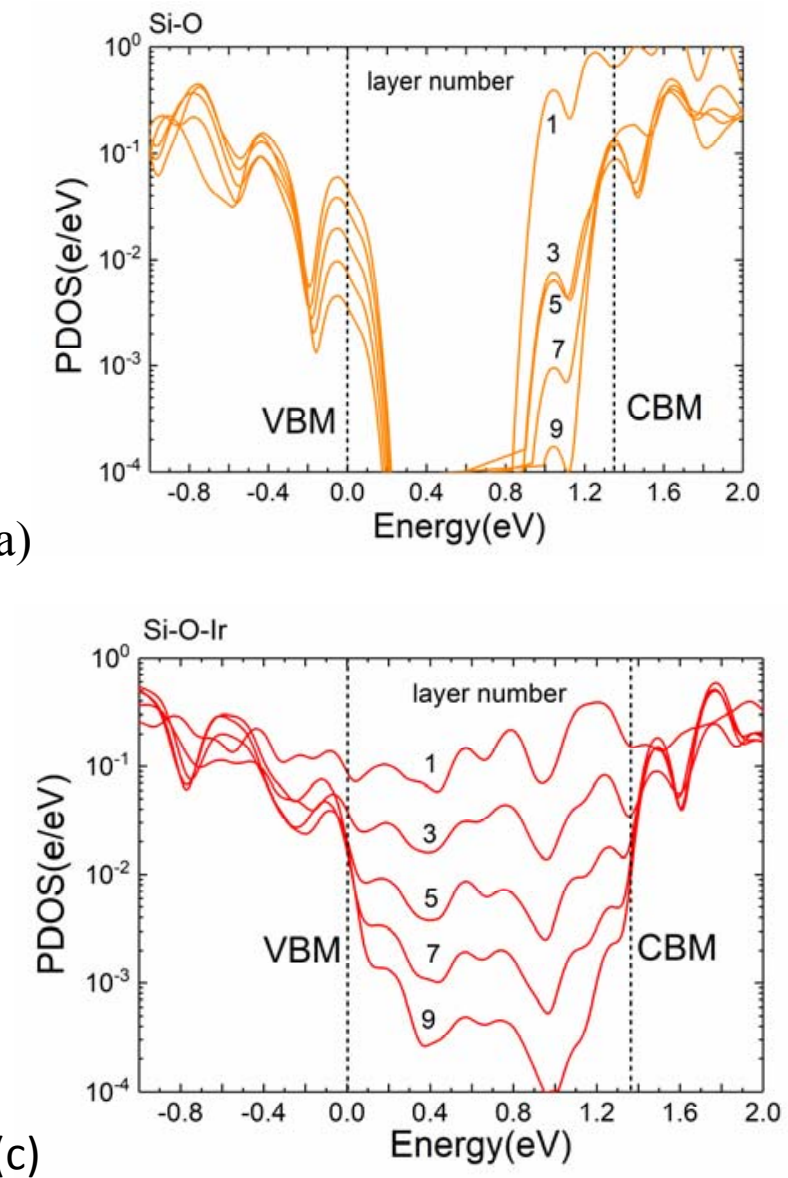

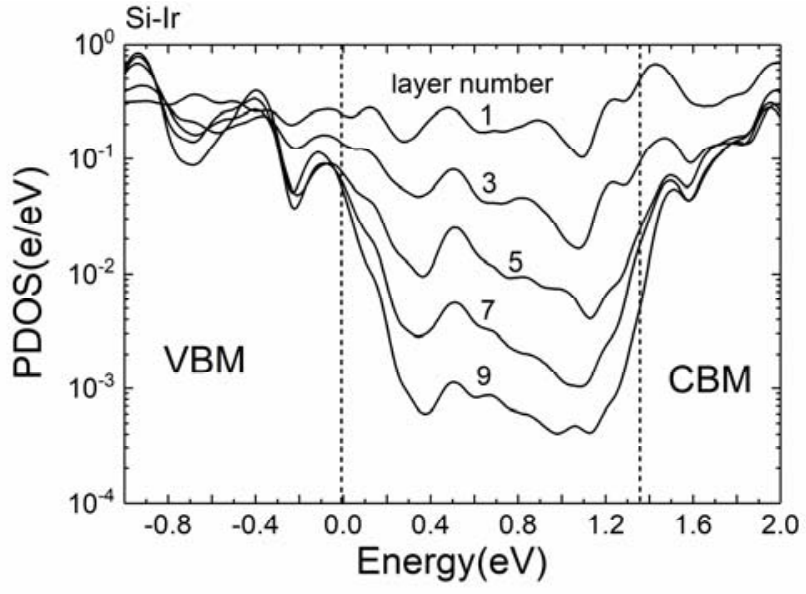

(b)

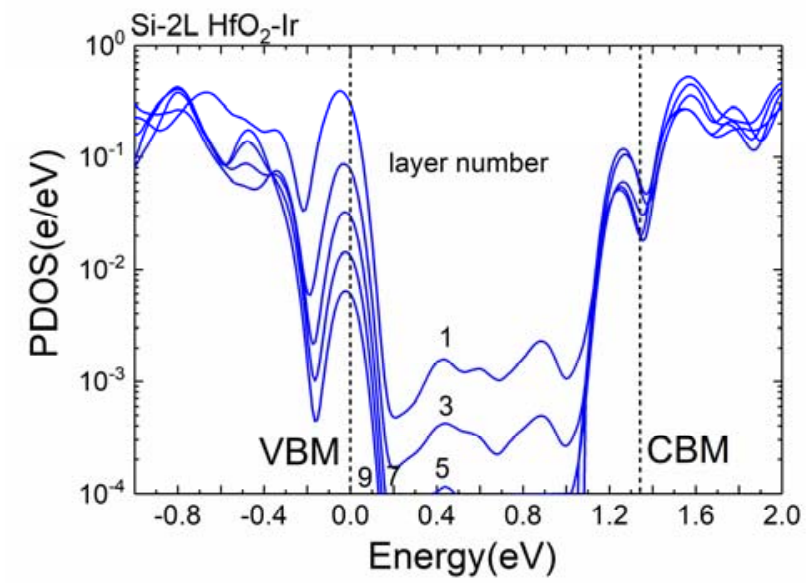

Fig. 3 


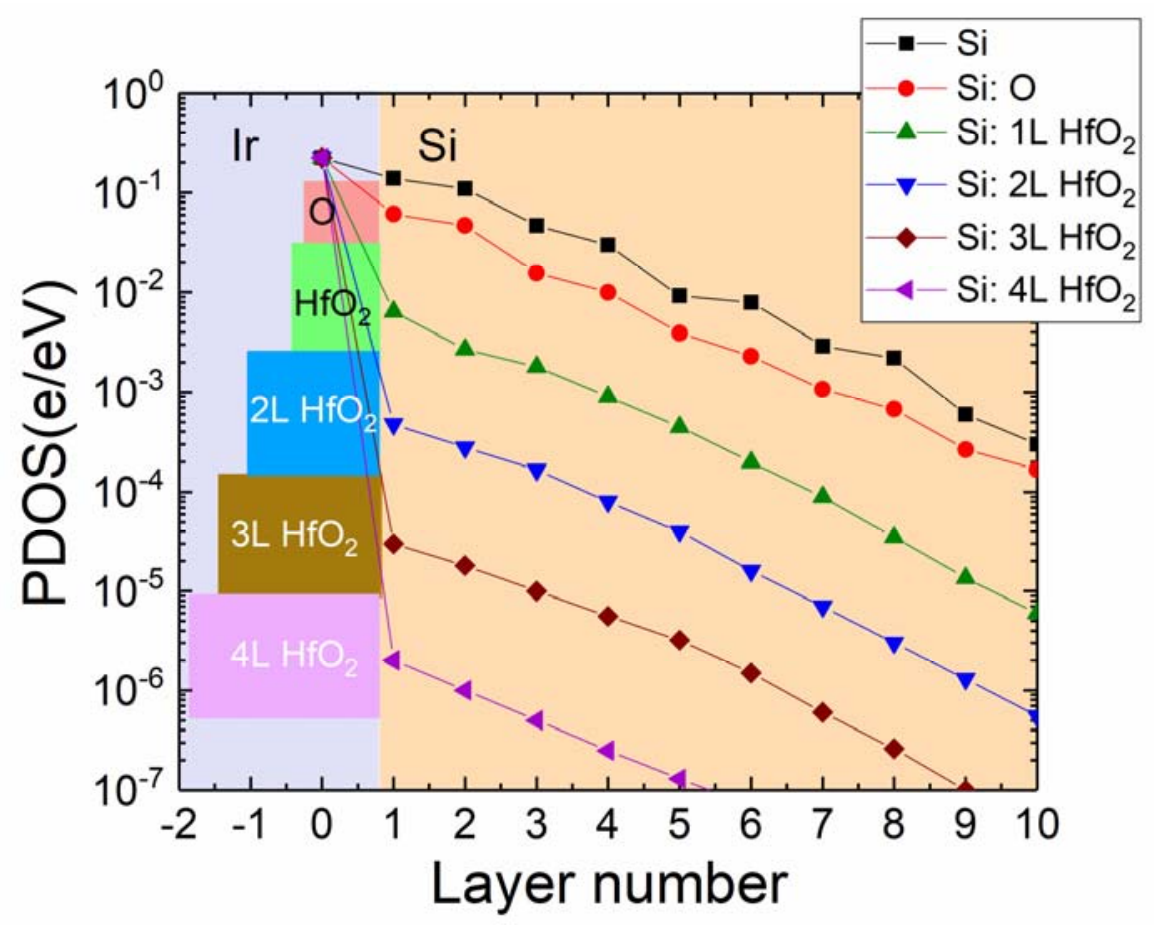

Fig. 4. 

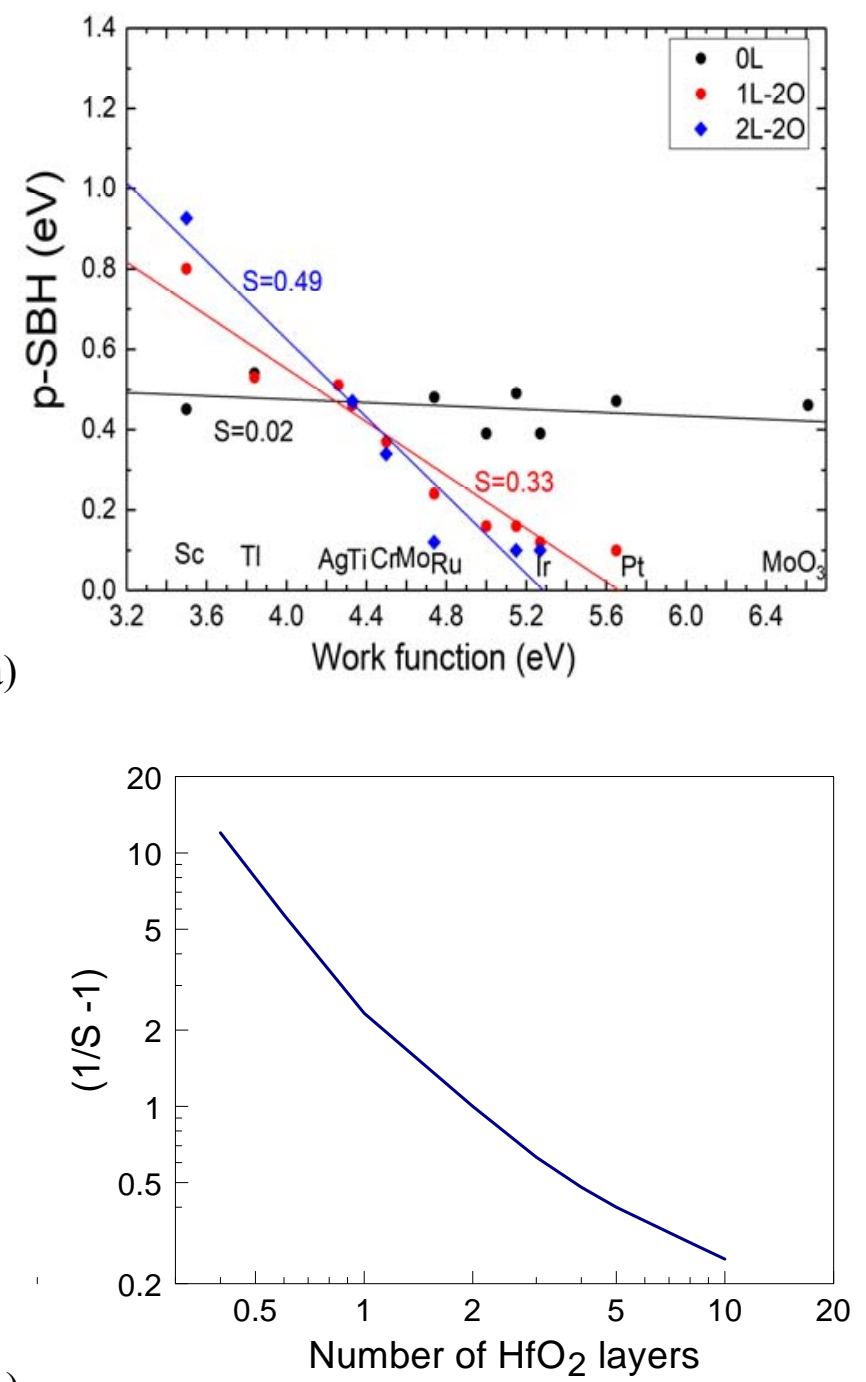

(b)

Fig. 5. 


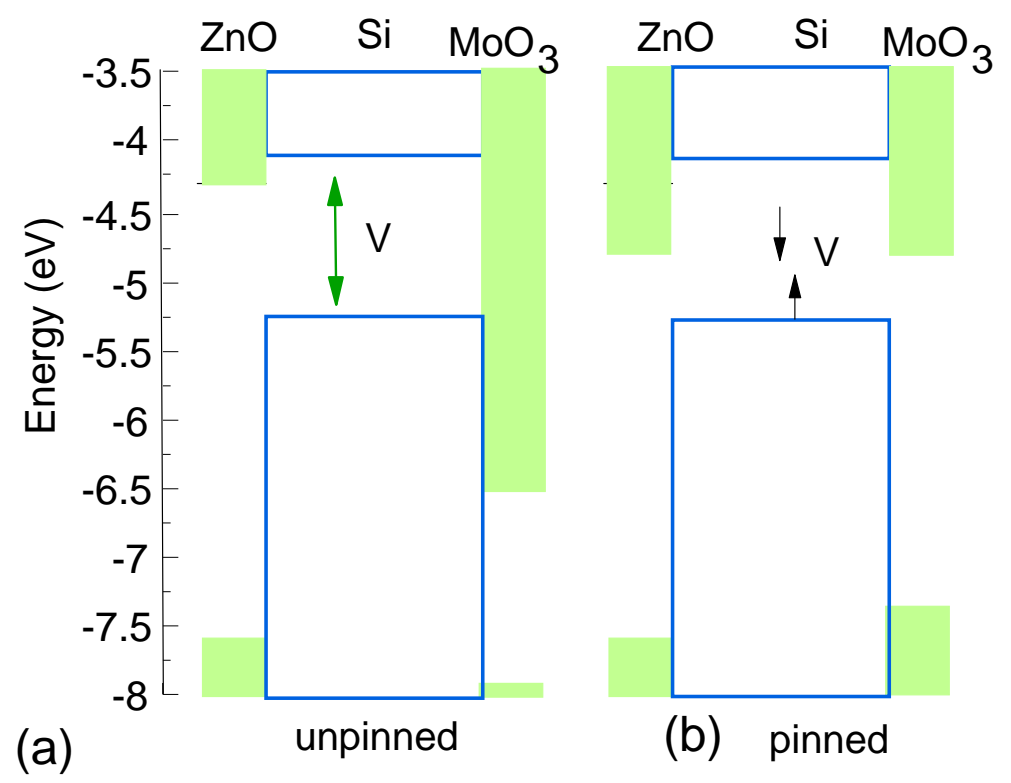

Fig.6. 